\title{
Effects of SGLT2 Inhibition on Diabetic Retinopathy
}

\author{
Sameer Leley ${ }^{1}$, Qianyi Luo ${ }^{2}$, Ashay Bhatwadekar ${ }^{2}$
}

${ }^{1}$ Indiana University School of Medicine; ${ }^{2}$ Department of Ophthalmology, Eugene and Marilyn Glick Eye Institute, Indiana University

\section{Background and Hypothesis:}

Diabetic retinopathy (DR), a microvascular complication of diabetes, is the leading cause of blindness in the working-age population, and its prevalence is increasing. New treatment modalities must be developed to slow the progression of DR. SGLT2 inhibition has shown promise in treating other diabetic complications; however, its effect on DR remains unknown, therefore, for this study, we hypothesize that SGLT2 inhibition will reduce the harmful effects of DR.

\section{Methods:}

Diabetic (db/db) mice were fed $10 \mathrm{mg} / \mathrm{kg}$ of the SGLT2 inhibitor dapagliflozin in their diet for 6 months, non-diabetic $(\mathrm{db} / \mathrm{m})$ mice on a regular diet served as controls. In parallel, human retinal endothelial cells (HREC) were used as in-vitro models and treated with dapagliflozin to assess glucose uptake via a 2-(N-(7-Nitrobenz-2-oxa-1,3-diazol-4-yl)Amino)-2-Deoxyglucose (2-NBDG) assay.

\section{Results:}

Our studies show that $\mathrm{db} / \mathrm{db}$ mice with dapagliflozin had significantly fewer acellular capillaries compared to untreated db/db mice. Furthermore, Dapagliflozin treatment at 1 and $10 \mu \mathrm{M}$ concentrations of dapagliflozin yielded a significant decrease in glucose uptake compared to respective vehicle controls.

\section{Conclusion:}

Our study shows that SGLT2 inhibition has a promise in treating DR by reducing acellular capillaries and retinal glucose transport suggesting the potential of dapagliflozin treatment in DR. 